\title{
Superadditive and subadditive transformations of integrals and aggregation functions
}

\author{
Salvatore Greco ${ }^{\star 12}$, Radko Mesiar ${ }^{\star \star 34}$, Fabio Rindone ${ }^{\star \star \star 1}$, and Ladislav Sipeky ${ }^{\dagger 3}$ \\ 1 Department of Economics and Business \\ 95029 Catania, Italy \\ 2 University of Portsmouth, Portsmouth Business School, Centre of Operations Research and \\ Logistics (CORL), Richmond Building, Portland Street, Portsmouth PO1 3DE, United Kingdom \\ ${ }^{3}$ Department of Mathematics and Descriptive Geometry, Faculty of Civil Engineering \\ Slovak University of Technology \\ 81005 Bratislava, Slovakia \\ 4 Institute of Theory of Information and Automation \\ Czech Academy of Sciences \\ Prague, Czech Republic
}

\begin{abstract}
We propose the concepts of superadditive and of subadditive transformations of aggregation functions acting on non-negative reals, in particular of integrals with respect to monotone measures. We discuss special properties of the proposed transforms and links between some distinguished integrals. Superadditive transformation of the Choquet integral, as well as of the Shilkret integral, is shown to coincide with the corresponding concave integral recently introduced by Lehrer. Similarly the transformation of the Sugeno integral is studied. Moreover, subadditive transformation of distinguished integrals is also discussed.
\end{abstract}

Keywords: Aggregation function; superadditive and subadditive transformations; fuzzy integrals.

\section{Introduction}

The concepts of subadditivity and superadditivity are very important in economics. For example, consider a production function $A: \mathbb{R}_{+}^{n} \rightarrow \mathbb{R}_{+}$assigning to each vector of production factors $\mathbf{x}=\left(x_{1}, \ldots, x_{n}\right)$ the corresponding output $A\left(x_{1}, \ldots, x_{n}\right)$. If one has available resources given by the vector $\overline{\mathbf{x}}=\left(\bar{x}_{1}, \ldots, \bar{x}_{n}\right)$, then, the production function $A$ assigns the output $A\left(\bar{x}_{1}, \ldots, \bar{x}_{n}\right)$. Now suppose that the resources $\overline{\mathbf{x}}=\left(\bar{x}_{1}, \ldots, \bar{x}_{n}\right)$ can be divided into $k \in \mathbb{N}$ subgroups of production factors $\overline{\mathbf{x}}=\left(\bar{x}_{1}, \ldots, \bar{x}_{n}\right)=$ $\left(x_{1}^{(1)}, \ldots, x_{n}^{(1)}\right)+\ldots+\left(x_{1}^{(k)}, \ldots, x_{n}^{(k)}\right)$. Since the purpose of any production function is to maximize the use of factor inputs in production, one should check if the production output $\sum_{i=1}^{k} A\left(x_{1}^{(i)}, \ldots, x_{n}^{(i)}\right)$ is greater than $A\left(\bar{x}_{1}, \ldots, \bar{x}_{n}\right)$. More in general, one

\footnotetext{
* salgreco@unict.it

** radko.mesiarestuba.sk

$\star \star \star$ frindonedunict.it

$\dagger$ ladislav.sipeky@stuba.sk
} 
can be interested in finding the "best" decomposition of the available resources, i.e., we should look to the quantity

$$
A^{*}(\overline{\mathbf{x}})=\sup \left\{\sum_{i=1}^{k} A\left(x_{1}^{(i)}, \ldots, x_{n}^{(i)}\right) \mid \sum_{i=1}^{k}\left(x_{1}^{(i)}, \ldots, x_{n}^{(i)}\right) \leq \overline{\mathbf{x}}\right\},
$$

provided that $\sum_{i=1}^{k}\left(x_{1}^{(i)}, \ldots, x_{n}^{(i)}\right)$ is an allowable (realistic) decomposition of $\overline{\boldsymbol{x}}$. Thus, either $A^{*}(\overline{\mathrm{x}})=A(\overline{\mathrm{x}})$ for all $\overline{\mathrm{x}} \in \mathbb{R}_{+}^{n}$ or function $A^{*}$ should be considered - at least ideally - the "real" production function (provided that the range of $A^{*}$ contains only finite values, i.e., $A^{*}(\overline{\boldsymbol{x}})<\infty$ for each $\left.\overline{\boldsymbol{x}} \in \mathbb{R}_{+}^{n}\right)^{5}$. The condition $A(\overline{\mathbf{x}})=A^{*}(\overline{\mathbf{x}})$ for all $\overline{\mathbf{x}} \in \mathbb{R}_{+}^{n}$ is equivalent to the superadditivity of the production function $A$, i.e., for all $\boldsymbol{y}, \boldsymbol{x} \in \mathbb{R}_{+}^{n}$ we have $A(\boldsymbol{y}+\boldsymbol{x}) \geq A(\boldsymbol{y})+A(\boldsymbol{x})$. Analogously, consider the situation of a system of prices represented by the function $A: \mathbb{R}_{+}^{n} \rightarrow \mathbb{R}_{+}$assigning to each bundle of goods $\mathbf{x}=\left(x_{1}, \ldots, x_{n}\right)$ with $x_{i}, i=1, \ldots, n$, representing the quantity of the $i$-th item, the corresponding price $A\left(x_{1}, \ldots, x_{n}\right)$. If one wants to buy the bundle $\overline{\mathbf{x}}=\left(\bar{x}_{1}, \ldots, \bar{x}_{n}\right)$, then one can get it at the following price (possibly asymptotically)

$$
A_{*}(\overline{\boldsymbol{x}})=\inf \left\{\sum_{i=1}^{k} A\left(x_{1}^{(i)}, \ldots, x_{n}^{(i)}\right) \mid \sum_{i=1}^{k}\left(x_{1}^{(i)}, \ldots, x_{n}^{(i)}\right) \geq \overline{\boldsymbol{x}}\right\} .
$$

Thus either $A(\overline{\mathbf{x}})=A_{*}(\overline{\mathbf{x}})$ for all $\overline{\mathbf{x}} \in \mathbb{R}_{+}^{n}$ or function $A_{*}$ becomes the "real" price system considered by economic operators. The condition $A(\overline{\mathbf{x}})=A_{*}(\overline{\mathbf{x}})$ for all $\overline{\mathbf{x}} \in \mathbb{R}_{+}^{n}$ is equivalent to the subadditivity of the price system $A$, i.e., for all $\boldsymbol{y}, \boldsymbol{x} \in \mathbb{R}_{+}^{n}$ we have $A(\boldsymbol{y}+\boldsymbol{x}) \leq A(\boldsymbol{y})+A(\boldsymbol{x})$

Observe that in two above examples the superadditivity and the subadditivity of function $A$ were related to its transformations $A^{*}$ and $A_{*}$, respectively. For this reason, it is important to study and discuss these transformations what we shall do in this paper.

For a class $\mathcal{K}$ of some objects, a property $\boldsymbol{p}$ determines a subclass

$$
\mathcal{K}_{\boldsymbol{p}}=\{K \in \mathcal{K} \mid K \text { has property } \boldsymbol{p}\} .
$$

Any mapping $\tau: \mathcal{K} \rightarrow \mathcal{K}$ is called a transformation (of objects from $\mathcal{K}$ ), and if $\mathcal{K}_{\tau}=$ $\{\tau(K) \mid K \in \mathcal{K}\}=\mathcal{K}_{p}$, and $\tau(K)=K$ for each $K \in \mathcal{K}_{p}, \tau$ is called a $\boldsymbol{p}$ transformation.

\footnotetext{
${ }^{5}$ To the best of our knowledge, this concept of transformation of the production function from $A$ to $A^{*}$ is original and not standard in the literature on production functions (see, e.g., [3]). Indeed according to Shephard [13] production function is defined as a relationship between the maximal technically feasible output and the inputs needed to produce that output, that corresponds to what we called "real production function" $A^{*}$. However, very often production function is simply defined as a technical relationship between output and inputs without any reference to the assumption that such output has to be maximal with respect to the given inputs (see [12]). In this sense, we can see that, when it is possible to imagine divisibility of the input, the superadditive transformation of the merely technical relationship between output and inputs $A$ gives the "real production function" $A^{*}$. Observe that in economics some assumptions are considered on production functions that imply their superadditivity. More precisely, continuity, strict increasing monotonicity, strict quasiconcavity and $A(0)=0$ are conditions usually assumed on production function. Under these conditions production function is superadditive ([13]; see also Theorem 3.1 in [6]).
} 
Obviously, $\tau \circ \tau=\tau$ for any $\boldsymbol{p}$-transformation $\tau$. Formally, $\tau$ can be seen as a projection from $\mathcal{K}$ onto $\mathcal{K}_{p}$.

We recall some typical examples:

- For $\mathcal{K}=\mathcal{M}_{\mathscr{S}}$ the class of monotone measures on a measurable space $(X, \mathscr{S})$, one can consider the superadditivity property. Define a transformation

$\tau: \mathcal{M}_{\mathscr{S}} \rightarrow \mathcal{M}_{\mathscr{S}}$ by $\tau(m): \mathscr{S} \rightarrow[0, \infty]$

$$
\tau(m)(E)=\sup \left\{\sum_{i=1}^{k} m\left(E_{i}\right) \mid\left(E_{i}\right)_{i=1}^{k} \text { is a measurable partition of } E\right\} .
$$

Observe that considering the PAN-integral $\int^{P A N}$ introduced in [18], see also [17], $\tau(m)(E)=\int^{P A N} \mathbf{1}_{E} d m$. It is not difficult to check that, taking the property $\boldsymbol{p}=$ superadditivity, then $\tau$ is a superadditive transformation.

- For $\mathcal{A}_{[0,1], n}$ the class of $n$-ary aggregation functions on $[0,1]$, one can consider the averaging property characterizing idempotent aggregation functions. Then, for the class $\mathcal{K} \subset \mathcal{A}_{[0,1], n}$ of $n$-ary aggregation functions with continuous strictly increasing diagonal section $\delta_{A}:[0,1] \rightarrow[0,1], \delta_{A}(x)=A(x, \ldots, x)$, the transformation $\tau: \mathcal{K} \rightarrow \mathcal{K}$ given by $\tau(A)\left(x_{1}, \ldots, x_{n}\right)=\delta_{A}^{-1}\left(A\left(x_{1}, \ldots, x_{n}\right)\right)$ (called the idempotization in [4]) is an averaging transformation.

Observe that the above mentioned transformations imply interesting consequences. For example, considering the superadditive transformation $\tau$ for any monotone measure $m \in \mathcal{M}_{\mathscr{S}}$, for any $\mathscr{S}$-measurable function $f: X \rightarrow[0, \infty]$, it holds

$$
\int^{P A N} f d m=\int^{P A N} f d \tau(m)
$$

and $\tau(m)$ is the smallest superadditive measure from $\mathcal{M}_{\mathscr{S}}$ bounded from below by $m$. On the other hand, considering the above mentioned averaging transformation $\tau$, for any strict triangular norm (in its $n$-ary form) $T:[0,1]^{n} \rightarrow[0,1]$ generated by an additive generator $f:[0,1] \rightarrow[0, \infty], \tau(T):[0,1]^{n} \rightarrow[0,1]$ is the related quasi-arithmetic mean generated by $f$,

$$
\tau(T)\left(x_{1}, \ldots, x_{n}\right)=f^{-1}\left(\frac{1}{n} \sum_{i-1}^{n} f\left(x_{i}\right)\right) .
$$

Based on $\tau$, one can generalize quasi-arithmetic means considering weakly increasing continuous t-norm $T:[0,1]^{n} \rightarrow[0,1]$, i.e., $T=\left(\left\langle a_{j}, b_{j}, T_{j}\right\rangle / j \in \mathcal{J}\right)$ is an ordinal sum with strict summands, for more details see [7]. If $f_{j}$ is an additive generator of $T_{j}, j \in \mathcal{J}$, then

$$
\tau(T)\left(x_{1}, \ldots, x_{n}\right)= \begin{cases}f_{j}^{-1}\left(\frac{1}{n} \sum_{i=1}^{n} f_{j}\left(\left|x_{i}\right|_{j}\right)\right) & \begin{array}{l}
\text { if } \left.\min \left\{x_{1}, \ldots, x_{n}\right\} \in\right] a_{j}, b_{j}[ \\
\text { for some } j \in \mathcal{J}
\end{array} \\
\min \left\{x_{1}, \ldots, x_{n}\right\} & \text { otherwise, }\end{cases}
$$


where $|x|_{j}=\min \left\{b_{j}, \max \left\{a_{j}, x\right\}\right\}$.

Our main aim is to study superadditive and subadditive transformations of aggregation functions acting on $\left[0, \infty\left[=\mathbb{R}_{+}\right.\right.$, in particular of some distinguished integrals. The paper is organized as follows. In next Section 2 we recall basic preliminaries concerning aggregation functions on $\mathbb{R}_{+}$, and in particular we recall some integrals here. Section 3 brings the proposal of a superadditive transformation including the study of its properties and some illustrative examples. In Section 4, a subadditive transformation is proposed and discussed. Section 5 is devoted to the examination of relationships between the superadditive transformations of some distinguished integrals. In Section 6, we discuss subadditive transformations of distinguished integrals. Note that here the role of the underlying measure is important to ensure the existence of subadditive transformations. Finally some concluding remarks are added.

\section{Aggregation functions on $\mathbb{R}_{+}$}

Let us consider the sets $N=\{1, \ldots, n\}$ and $\mathbb{R}_{+}=\left[0,+\infty\left[\right.\right.$. For all $E \subseteq N, \mathbf{1}_{E}$ is the vector of $\mathbb{R}_{+}^{n}$ whose $i$ th component equals 1 if $i \in E$ and equals 0 otherwise. $\mathbf{0}=(0, \ldots, 0)$ indicates the null vector.

An aggregation function $A$ on $\mathbb{R}_{+}^{n}$ is a monotone function $A: \mathbb{R}_{+}^{n} \rightarrow \mathbb{R}_{+}$such that $A(\mathbf{0})=0$ and $\sup _{\boldsymbol{x} \in \mathbb{R}_{+}^{n}} A(\boldsymbol{x})>0$, [4]. Note that we have relaxed the constraint $\sup _{\boldsymbol{x} \in \mathbb{R}_{+}^{n}} A(\boldsymbol{x})=+\infty$ considered in [4] to cover integrals considered in this paper, in particular the Sugeno integral [16].

An aggregation function $A$ on $\mathbb{R}_{+}^{n}$ is said to be

- idempotent, if for all $\lambda \in \mathbb{R}_{+}, A\left(\lambda \mathbf{1}_{N}\right)=\lambda$;

- homogeneous, if for all $\lambda>0$ and for all $\boldsymbol{x} \in \mathbb{R}_{+}^{n}, A(\lambda \boldsymbol{x})=\lambda A(\boldsymbol{x})$;

- concave (convex), if for all for all $\boldsymbol{x}, \boldsymbol{y} \in \mathbb{R}_{+}^{n}$ and for all $\lambda \in[0,1]$,

$$
\begin{gathered}
A(\lambda \boldsymbol{x}+(1-\lambda) \boldsymbol{y}) \geq \lambda A(\boldsymbol{x})+(1-\lambda) A(\boldsymbol{y}) \\
[A \lambda \boldsymbol{x}+(1-\lambda) \boldsymbol{y}) \leq \lambda A(\boldsymbol{x})+(1-\lambda) A(\boldsymbol{y})] ;
\end{gathered}
$$

- superadditive, (subadditive) if for all $\boldsymbol{x}, \boldsymbol{y} \in \mathbb{R}_{+}^{n}$

$$
A(\boldsymbol{x})+A(\boldsymbol{y}) \leq A(\boldsymbol{x}+\boldsymbol{y}) \quad[A(\boldsymbol{x})+A(\boldsymbol{y}) \geq A(\boldsymbol{x}+\boldsymbol{y})] .
$$

An aggregation function $A$ being superadditive (subadditive) and homogeneous is called superlinear (sublinear). Observe that any superlinear (sublinear) aggregation function is concave (convex), while the inverse is not true (e.g., $f(x)=\sqrt{x}$ is concave but it neither homogeneous nor superadditive). In fact, for homogeneous aggregation function on $\mathbb{R}_{+}^{n}$, the concavity (convexity) is equivalent to the superadditivity (subadditivity).

Typical examples of aggregation functions on $\mathbb{R}_{+}^{n}$ are:

- weighted sums, $W\left(x_{1}, \ldots, x_{n}\right)=\sum_{i=1}^{n} w_{i} x_{i}$, with weights $w_{i} \in \mathbb{R}_{+}$and at least one non-zero weight; these aggregation functions satisfy all above mentioned properties (and they are unique with these properties); 
- the smallest idempotent aggregation function min, $\min \left(x_{1}, \ldots, x_{n}\right)=\min \left\{x_{1}, \ldots, x_{n}\right\}$, is homogeneous, superadditive, concave, and superlinear;

- the greatest idempotent aggregation function $M a x$, $\operatorname{Max}\left(x_{1}, \ldots, x_{n}\right)=\max \left\{x_{1}, \ldots, x_{n}\right\}$, is homogeneous, subadditive, convex, and sublinear;

- the geometric mean $G, G\left(x_{1}, \ldots, x_{n}\right)=\sqrt[n]{\prod_{i=1}^{n} x_{i}}$, is idempotent, homogeneous, superadditive, concave, and superlinear;

- the quadratic mean $Q, Q\left(x_{1}, \ldots, x_{n}\right)=\sqrt{\frac{1}{n} \sum_{i=1}^{n} x_{i}^{2}}$, is idempotent, homogeneous, convex, and sublinear;

- define $D: \mathbb{R}_{+}^{2} \rightarrow \mathbb{R}_{+}$by $D(x, y)=x^{2}+\sqrt{y}$. Then $D$ is a binary aggregation function which has none of the above mentioned properties.

Typical aggregation functions are integrals. Definitions of integrals are based on special set-functions, namely measures. A measure $\mu$ on $N$ is a monotone (w.r.t. set inclusion) function $\mu: 2^{N} \rightarrow \mathbb{R}_{+}$satisfying the condition $\mu(\emptyset)=0$ and $\mu(N)>0$.

The Choquet integral [2] of $\boldsymbol{x} \in \mathbb{R}_{+}^{n}$ with respect to the measure $\mu$ is defined as:

$$
\int^{C h} \boldsymbol{x} d \mu=\int_{0}^{\max _{i \in N} x_{i}} \mu\left(\left\{i \in N: x_{i} \geq t\right\}\right) d t .
$$

The Sugeno integral [16] of $\boldsymbol{x} \in \mathbb{R}_{+}^{n}$ with respect to the measure $\mu$ is defined as:

$$
\int^{S u} \boldsymbol{x} d \mu=\max \left\{\min \left\{x_{i}, \mu\left(\left\{j \in N: x_{j} \geq x_{i}\right\}\right)\right\}: i=1, \ldots, n\right\} .
$$

The Shilkret integral [14] of $\boldsymbol{x} \in \mathbb{R}_{+}^{n}$ with respect to the measure $\mu$ is defined as:

$$
\int^{S h} \boldsymbol{x} d \mu=\max \left\{x_{i} \mu\left(\left\{j \in N: x_{j} \geq x_{i}\right\}\right): i=1, \ldots, n\right\} .
$$

The concave integral [9] of $\boldsymbol{x} \in \mathbb{R}_{+}^{n}$ with respect to the measure $\mu$ is defined as:

$$
\int^{c a v} \boldsymbol{x} d \mu=\sup \left\{\sum_{T \subseteq N} \alpha_{T} \mu(T): \sum_{T \subseteq N} \alpha_{T} \mathbf{1}_{T} \leq \boldsymbol{x}, \alpha_{T} \geq 0 \text { for all } T \subseteq N\right\} .
$$

The convex integral [10] of $\boldsymbol{x} \in \mathbb{R}_{+}^{n}$ with respect to the measure $\mu$ is defined as:

$$
\int^{c o n} \boldsymbol{x} d \mu=\inf \left\{\sum_{T \subseteq N} \alpha_{T} \mu(T): \sum_{T \subseteq N} \alpha_{T} \mathbf{1}_{T} \geq \boldsymbol{x}, \alpha_{T} \geq 0 \text { for all } T \subseteq N\right\} .
$$

The PAN integral [17] of $\boldsymbol{x} \in \mathbb{R}_{+}^{n}$ with respect to the measure $\mu$ is defined as:

$$
\int^{P A N} \boldsymbol{x} d \mu=\sup \left\{\sum_{j=1}^{k} a_{j} \mu\left(A_{j}\right): \sum_{j=1}^{k} a_{j} \mathbf{1}_{A_{j}} \leq \boldsymbol{x},\left\{A_{j}\right\}_{1}^{k} \text { being a partition of } N\right\} .
$$


The downward PAN integral of $\boldsymbol{x} \in \mathbb{R}_{+}^{n}$ with respect to the measure $\mu$ is defined as:

$$
\int^{P A N \downarrow} \boldsymbol{x} d \mu=\inf \left\{\sum_{j=1}^{k} a_{j} \mu\left(A_{j}\right): \sum_{j=1}^{k} a_{j} \mathbf{1}_{A_{j}} \geq \boldsymbol{x},\left\{A_{j}\right\}_{1}^{k} \text { being a partition of } N\right\} .
$$

\section{Superadditive transformations of aggregation functions}

Definition 1 Given an aggregation function $A$ on $\mathbb{R}_{+}^{n}$ and $\boldsymbol{x} \in \mathbb{R}_{+}^{n}$, if

$$
A^{*}(\boldsymbol{x}):=\sup \left\{\sum_{j=1}^{k} A\left(\boldsymbol{y}^{j}\right): \boldsymbol{y}^{j} \in \mathbb{R}_{+}^{n} \text { such that } \sum_{j=1}^{k} \boldsymbol{y}^{j} \leq \boldsymbol{x}\right\}<+\infty, \text { (Fin) }
$$

then $A^{*}(\boldsymbol{x})$ is called a superadditive transformation of $\boldsymbol{x}$ with respect to $A$.

Let us note that, in definition 1, when looking for the supremum of all possible sums $\sum_{j=1}^{k} A\left(\boldsymbol{y}^{j}\right)\left(\sum_{j=1}^{k} \boldsymbol{y}^{j}\right.$ being not greater than $\left.\boldsymbol{x}\right)$, the integer $k$ is not fixed.

In general, the symbol $A^{*}(\boldsymbol{x})$ indicates the sup in condition (Fin) and, by definition, for any $\boldsymbol{x}, \boldsymbol{y} \in \mathbb{R}_{+}^{n}$, if $\boldsymbol{y} \leq \boldsymbol{x}$, then $A^{*}(\boldsymbol{y}) \leq A^{*}(\boldsymbol{x}) \leq+\infty$.

We say that an aggregation function $A: \mathbb{R}_{+}^{n} \rightarrow \mathbb{R}_{+}$admits superadditive transformation on $\mathbb{R}_{+}^{n}$ if the condition (Fin) is satisfied for all $\boldsymbol{x} \in \mathbb{R}_{+}^{n}$. Denote by $\mathcal{K}_{n}^{*}$ the class of all aggregation functions which admit superadditive transformation on $\mathbb{R}_{+}^{n}$.

Theorem 1 Let $A: \mathbb{R}_{+}^{n} \rightarrow \mathbb{R}_{+}$be an aggregation function. Then $A \in \mathcal{K}_{n}^{*}$, i.e.,

$$
\sup \left\{\sum_{j=1}^{k} A\left(\boldsymbol{y}^{j}\right): \boldsymbol{y}^{j} \in \mathbb{R}_{+}^{n} \text { such that } \sum_{j=1}^{k} \boldsymbol{y}^{j} \leq \boldsymbol{x}\right\}<+\infty, \forall \boldsymbol{x} \in \mathbb{R}_{+}^{n}
$$

if and only if

$$
A^{*}\left(\mathbf{1}_{N}\right)=\sup \left\{\sum_{j=1}^{k} A\left(\boldsymbol{y}^{j}\right): \boldsymbol{y}^{j} \in \mathbb{R}_{+}^{n} \text { such that } \sum_{j=1}^{k} \boldsymbol{y}^{j} \leq \mathbf{1}_{N}\right\}<+\infty .
$$

In other words, a necessary and sufficient condition ensuring that A admits superadditive extension on $\mathbb{R}_{+}^{n}$ is that (Fin) holds for the constant vector $\mathbf{1}_{N}$.

Proof. Obviously, we need only to prove the sufficiency. By the monotonicity of $A^{*}$ it is sufficient to prove that $A^{*}(\boldsymbol{x})<\infty$ for any $\boldsymbol{x} \in \mathbb{N}^{n}$. Define $|\boldsymbol{x}|=\sum_{i=1}^{n} x_{i}$ for any $\boldsymbol{x} \in \mathbb{N}^{n}$, and put $K_{m}=\max \left\{A^{*}(\boldsymbol{x})\left|\boldsymbol{x} \in \mathbb{N}^{n},\right| \boldsymbol{x} \mid=m\right\}$. The proof is complete if we show that $K_{m}<\infty$ for any $m \in \mathbb{N}$. We prove this statement by induction. By the assumption, $K_{1}<\infty$. Let $K_{m}<\infty$ (induction step), and let $\boldsymbol{x} \in \mathbb{N}^{n}$ such that $|\boldsymbol{x}|=m+1$. If $x_{i} \leq 1$ for any $i=1, \ldots, n$, then $A^{*}(\boldsymbol{x}) \leq A^{*}\left(\mathbf{1}_{N}\right)<\infty$. If $x_{i}>1$ for some $i=1, \ldots, n$, put $\boldsymbol{x}^{\prime}=\left(x_{1}, \ldots, x_{i}-1, \ldots, x_{n}\right)$. Let $\sum_{j=1}^{k} \boldsymbol{y}^{j}=\boldsymbol{x}$ (from the monotonicity it is sufficient to consider the equality). Then there exists $0 \leq l \leq k$ such 
that $\sum_{j=1}^{l} \boldsymbol{y}^{j} \leq \boldsymbol{x}^{\prime}$ and $\sum_{j=1}^{l+1} \boldsymbol{y}^{j}>\boldsymbol{x}^{\prime}$, where we consider the sum over the empty index set to be equal to 0 . Then, we can write

$$
\sum_{j=1}^{k} \boldsymbol{y}^{j}=\sum_{j=1}^{l} \boldsymbol{y}^{j}+\boldsymbol{y}^{l+1}+\sum_{j=l+2}^{k} \boldsymbol{y}^{j}
$$

where we admit that some of the sums on the right side of the equality can have the empty index set (this is the case if $l=0$ or $l=k-1$ ). Since $\sum_{j=1}^{l} \boldsymbol{y}^{j} \leq \boldsymbol{x}^{\prime}$ and also $\sum_{j=l+2}^{k} \boldsymbol{y}^{j} \leq \boldsymbol{x}^{\prime}$, we simply find that

$$
\sum_{j=1}^{k} A\left(\boldsymbol{y}^{j}\right) \leq 2 A^{*}\left(\boldsymbol{x}^{\prime}\right)+A(\boldsymbol{x}) \leq 2 K_{m}+A(\boldsymbol{x}) .
$$

Then, $A^{*}(\boldsymbol{x}) \leq 2 K_{m}+A(\boldsymbol{x})<\infty$ by the induction step $K_{m}<\infty$ and the fact that $A(\boldsymbol{x})<\infty$. Hence, from the previous result, we obtain that $A^{*}(\boldsymbol{x})<\infty$ for any $\boldsymbol{x} \in \mathbb{N}^{n}$ such that $|\boldsymbol{x}|=m+1$, which implies $K_{m+1}<\infty$.

Observe that the input $\mathbf{1}_{N}$ in Theorem 1 can be replaced by an arbitrary vector $\boldsymbol{x}$ from $\mathbb{R}_{+}^{n}$ with full support (i.e., $x_{i}>0$ for $i=1, \ldots, n$ ), and in particular by $\varepsilon \mathbf{1}_{N}$ with $\varepsilon$ arbitrarily small but positive. Based on Theorem 1, also the next result, dealing directly with the underlying aggregation function $A$, can be introduced.

Theorem 2 An aggregation function $A: \mathbb{R}_{+}^{n} \rightarrow \mathbb{R}_{+}$is an element of $\mathcal{K}_{n}^{*}$ if and only if there is a constant $p \in] 0, \infty[$ such that $A(x, \ldots, x) \leq p \cdot x$ for all $x \in[0,1]$.

Proof. For the sufficiency, due to Theorem 1, it is enough to show that $A^{*}\left(\mathbf{1}_{N}\right)$ is finite. Due to the monotonicity of $A$, it is obvious that for any $\boldsymbol{y}=\left(y_{1}, \ldots, y_{n}\right)$ from $[0,1]^{n}$ it holds $A(\boldsymbol{y}) \leq p \cdot \sum_{i=1}^{n} y_{i}$. Therefore, if $\sum_{j=1}^{k} \boldsymbol{y}^{j} \leq \mathbf{1}_{N}$ then

$$
\sum_{j=1}^{k} A\left(\boldsymbol{y}^{j}\right) \leq \sum_{j=1}^{k} p \cdot \sum_{i=1}^{n} y_{i}^{j} \leq n \cdot p
$$

what ensures $A^{*}\left(\mathbf{1}_{N}\right) \leq n \cdot p<\infty$.

To see the necessity, suppose that for each $p \in] 0, \infty[$ there is $x \in] 0,1]$ such that $A(x, \ldots, x)>p \cdot x$. Then, there is a sequence $\left(x_{m}\right)_{m \in \mathbb{N}}$ such that $A\left(x_{m}, \ldots, x_{m}\right)>$ $m \cdot x_{m}$, for any $m \in \mathbb{N}$. If $\left[\frac{1}{x_{m}}\right]$ is the integer part of $\frac{1}{x_{m}}$ we have that for each $m \in \mathbb{N}, A^{*}\left(1_{N}\right)+A\left(1_{N}\right) \geq A^{*}\left(1_{N}\right)+A\left(x_{m}, \ldots, x_{m}\right) \geq A^{*}\left(\left[\frac{1}{x_{m}}\right] x_{m} \cdot 1_{N}\right)+$ $A\left(x_{m}, \ldots, x_{m}\right) \geq\left[\frac{1}{x_{m}}\right] A\left(x_{m}, \ldots, x_{m}\right)+A\left(x_{m}, \ldots, x_{m}\right)>\left(\left[\frac{1}{x_{m}}\right]+1\right) x_{m} \cdot m \geq m$, and then $A^{*}\left(1_{N}\right)>m-A\left(1_{N}\right)$ for all $m \in \mathbb{N}$, i.e., $A^{*}\left(1_{N}\right)=\infty$. Due to Theorem 1 , $A \notin \mathcal{K}_{n}^{*}$. 
Remark 1 Note that the interval $[0,1]$ considered in Theorem 2 can be replaced by an interval $[0, \varepsilon]$ with $\varepsilon>0$ arbitrarily small, i.e., the properties of an aggregation function $A$ related to its superadditive extendibility depends on the properties of $A$ in some neighborhood of point $(0, \ldots, 0)$.

Remark 2 Observe that by Theorem 2 we get that if the Lipschitz condition holds in a neighborhood of $(0, \ldots, 0)$ with positive Lebesgue measure, then $A \in \mathcal{K}_{n}^{*}$.

Next corollaries individuate large classes of aggregation functions admitting superadditive extension.

Corollary 1 Let $A: \mathbb{R}_{+}^{n} \rightarrow \mathbb{R}_{+}$be an aggregation function such that there exists $k>0$ for which $A(\boldsymbol{x}) \leq k \sum_{i=1}^{n} x_{i}$ holds for any $\boldsymbol{x}=\left(x_{1}, \ldots, x_{n}\right) \in \mathbb{R}_{+}^{n}$. Then, $A \in \mathcal{K}_{n}^{*}$.

Corollary 2 Let $A: \mathbb{R}_{+}^{n} \rightarrow \mathbb{R}_{+}$be an aggregation function such that there exists $k>0$ for which $A(\boldsymbol{x}) \leq k \cdot \max _{i} x_{i}$ holds for any $\boldsymbol{x}=\left(x_{1}, \ldots, x_{n}\right) \in \mathbb{R}_{+}^{n}$. Then, $A \in \mathcal{K}_{n}^{*}$.

Corollary 3 Let $A: \mathbb{R}_{+}^{n} \rightarrow \mathbb{R}_{+}$be an aggregation function such that $A / k$ is idempotent for some $k \in] 0, \infty\left[\right.$. Then $A \in \mathcal{K}_{n}^{*}$.

The proof of previous corollaries is not difficult: Corollary 1 follows from Theorem 2 , considering the constant $n \cdot k$; Corollary 2 follows from Corollary 1 , and Corollary 3 follows from Theorem 2 , where we require that $A / p$ is subidempotent on $[0,1]$.

Now we give some examples of aggregation function which do not satisfy condition $(F i n)$. This is the case of any aggregation function which is non continuous in $\mathbf{0}=$ $(0, \ldots, 0)$. For example, let us consider the aggregation function $A: \mathbb{R}_{+}^{n} \rightarrow \mathbb{R}_{+}$defined for all $\boldsymbol{x}=\left(x_{1}, \ldots, x_{n}\right) \in \mathbb{R}_{+}^{n}$ by

$$
A(\boldsymbol{x})= \begin{cases}0 & \text { if } x_{i}=0 \text { for all } i=1, \ldots, n \\ \max _{i} x_{i}+1 & \text { else. }\end{cases}
$$

Since we can decompose vector $\mathbf{1}_{N}$ in a denumerable sum $\mathbf{1}_{N}=\sum_{k=1}^{\infty}\left(\frac{1}{2^{k}}, \ldots, \frac{1}{2^{k}}\right)$ and since $A\left(\frac{1}{2^{k}}, \ldots, \frac{1}{2^{k}}\right)=\frac{1}{2^{k}}+1$, then we can make the $\operatorname{sum} \sum_{k=1}^{n} A\left(\frac{1}{2^{k}}, \ldots, \frac{1}{2^{k}}\right)$ as large as desired.

Another class of aggregation functions which do not satisfy condition (Fin) is given by aggregation function with partial derivatives which are not bounded in neighborhood of $\mathbf{0}=(0, \ldots, 0)$, such as $A\left(x_{1}, \ldots, x_{n}\right)=\sum_{i=1}^{n} \sqrt{x_{i}}$.

Proposition 1 For any aggregation function $A$ on $\mathbb{R}_{+}^{n}, A \in \mathcal{K}_{n}^{*}$, it holds

- $A^{*}(\boldsymbol{x}) \geq A(\boldsymbol{x})$, for all $\boldsymbol{x} \in \mathbb{R}_{+}^{n}$,

- $A^{*}(\mathbf{0})=0$, and

- $A^{*}(\boldsymbol{x}) \geq A^{*}(\boldsymbol{y})$ for all $\boldsymbol{x}, \boldsymbol{y} \in \mathbb{R}_{+}^{n}$ such that $\boldsymbol{x} \geq \boldsymbol{y}$, i.e., $A^{*}$ is an aggregation function on $\mathbb{R}_{+}^{n}$ and $A^{*} \geq A$.

Proof. The proof follows directly from the definition of the function $A^{*}$ when considering $A \in \mathcal{K}_{n}^{*}$ 
Proposition 2 Let $A \in \mathcal{K}_{n}^{*}$. Then $A^{*}$ is a superadditive aggregation function on $\mathbb{R}_{+}^{n}$. Moreover

$$
A^{*}(\boldsymbol{x})=\min \left\{C(\boldsymbol{x}): C(\boldsymbol{y}) \geq A(\boldsymbol{y}), \forall \boldsymbol{y} \in \mathbb{R}_{+}^{n}\right\},
$$

where the minimum is taken over all the superadditive aggregation functions on $\mathbb{R}_{+}^{n}$ bounded from below by $A$.

Proof. By Proposition $1, A^{*}$ is an aggregation function. For any $\boldsymbol{x}, \boldsymbol{y} \in \mathbb{R}_{+}^{n}$, if $\sum_{j=1}^{k} \boldsymbol{u}^{j} \leq \boldsymbol{x}$ and $\sum_{j=1}^{p} \boldsymbol{v}^{j} \leq \boldsymbol{y}$, then $\sum_{j=1}^{k} \boldsymbol{u}^{j}+\sum_{j=1}^{k} \boldsymbol{v}^{j} \leq \boldsymbol{x}+\boldsymbol{y}$, and thus $A^{*}(\boldsymbol{x})+A^{*}(\boldsymbol{y})=$ $=\sup \left\{\sum_{j=1}^{k} A\left(\boldsymbol{u}^{j}\right) \mid \sum_{j=1}^{k} \boldsymbol{u}^{j} \leq \boldsymbol{x}\right\}+\sup \left\{\sum_{j=1}^{p} A\left(\boldsymbol{v}^{j}\right) \mid \sum_{j=1}^{p} \boldsymbol{v}^{j} \leq \boldsymbol{y}\right\}=$ $=\sup \left\{\sum_{j=1}^{k} A\left(\boldsymbol{u}^{j}\right)+\sum_{j=1}^{p} A\left(\boldsymbol{v}^{j}\right) \mid \sum_{j=1}^{k} \boldsymbol{u}^{j} \leq \boldsymbol{x}\right.$ and $\left.\sum_{j=1}^{p} \boldsymbol{v}^{j} \leq \boldsymbol{y}\right\} \leq$ $\leq \sup \left\{\sum_{j=1}^{r} A\left(\boldsymbol{z}^{j}\right) \mid \sum_{j=1}^{r} \boldsymbol{z}^{j} \leq \boldsymbol{x}+\boldsymbol{y}\right\}=A^{*}(\boldsymbol{x}+\boldsymbol{y})$, proving the superadditivity of $A^{*}$.

Let $C: \mathbb{R}_{+}^{n} \rightarrow \mathbb{R}_{+}$be a superadditive aggregation function with $C(\boldsymbol{x}) \geq A(\boldsymbol{x})$ for all $\boldsymbol{x} \in \mathbb{R}_{+}^{n}$. Let $\boldsymbol{x}$ be a vector from $\mathbb{R}_{+}^{n}$. For each decomposition $\sum_{i=1}^{k} \boldsymbol{x}^{i}=\boldsymbol{x}$, with $\boldsymbol{x}^{1}, \ldots, \boldsymbol{x}^{k} \in \mathbb{R}_{+}^{n}$ we have that $\sum_{i=1}^{k} A\left(\boldsymbol{x}^{i}\right) \leq \sum_{i=1}^{k} C\left(\boldsymbol{x}^{i}\right) \leq C\left(\sum_{i=1}^{k} \boldsymbol{x}^{i}\right)=C(\boldsymbol{x})$, and then $C(\boldsymbol{x})$ is an upper bound of the set whose $A^{*}(\boldsymbol{x})$ is the supremum, which implies $A^{*}(\boldsymbol{x}) \leq C(\boldsymbol{x})$.

Now, the superadditivity of $A^{*}$ yields the desired result, that $A^{*}(\boldsymbol{x})=\min \left\{C(\boldsymbol{x}): C \geq A, C\right.$ is superadditive aggregation function on $\left.\mathbb{R}_{+}^{n}\right\}$.

Observe that if $A \notin \mathcal{K}_{n}^{*}$ then there is no superadditive aggregation function $B$ on $\mathbb{R}_{+}^{n}$ dominating $A$ (i.e., $B \geq A$ ). Equivalently, elements of $\mathcal{K}_{n}^{*}$ are just aggregation functions on $\mathbb{R}_{+}^{n}$ admitting a dominating superadditive aggregation function on $\mathbb{R}_{+}^{n}$. Note also that for any $A \in \mathcal{K}_{n}^{*}, A^{*}$ is an aggregation function in the sense of [4], i.e., $\sup _{x \in \mathbb{R}} A^{*}(\boldsymbol{x})=+\infty$.

Now we are ready to introduce a superadditive transformation of aggregation functions from $\mathcal{K}_{n}^{*}$.

Theorem 3 Define a mapping $\tau^{*}: \mathcal{K}_{n}^{*} \rightarrow \mathcal{K}_{n}^{*}$ by $\tau^{*}(A)=A^{*}$. Then $\tau^{*}$ is a superadditive transformation.

Proof. Due to Proposition $1, \tau^{*}(A)=A^{*}$ is an aggregation function on $\mathbb{R}_{+}^{n}$ for any $A \in \mathcal{K}_{n}^{*}$. Moreover, based on the ideas in the proof of Theorem 1 , it is not difficult to check that for each $A \in \mathcal{K}_{n}^{*},\left(A^{*}\right)^{*}\left(\mathbf{1}_{N}\right)$ is finite and hence $A^{*} \in \mathcal{K}_{n}^{*}$, showing that the transformation $\tau^{*}$ is well defined. Finally, for any superadditive $A \in \mathcal{K}_{n}^{*}$, by induction we have $A\left(\sum_{j=1}^{k} \boldsymbol{y}^{j}\right) \geq \sum_{j=1}^{k} A\left(\boldsymbol{y}^{j}\right)$, and thus $\tau^{*}(A)=A^{*}=A$.

We list now some additional properties and examples of the introduced superadditive transformation. 
- $A$ is superadditive if and only if $A^{*}=A$;

- $A^{* *}=A^{*}$;

- if $A \geq B$ then $A^{*} \geq B^{*}$;

- if $A \geq B \geq C$ and $A^{*}=C^{*}$, then $B^{*}=A^{*}$;

- if $A \geq B \geq D, A \geq C \geq D$, and $A^{*}=D^{*}$, then $(\lambda B+(1-\lambda) C)^{*}=A^{*}$ for all $\lambda \in[0,1]$;

- if $A=\min$, then $A^{*}=A$ (observe that Minimum is superadditive);

- if $A=$ Max, then $A^{*}(\mathbf{x})=\sum_{i=1}^{n} x_{i}$; moreover, for any $A$ between max and sum, we have that $A^{*}$ is the sum;

- if $A$ is modular, i.e., if $A(\mathbf{x})=\sum_{i=1}^{n} f_{i}\left(x_{i}\right)$ then $A^{*}$ is modular, $A^{*}(\mathbf{x})=\sum_{i=1}^{n} f_{i}^{*}\left(x_{i}\right)$, where for $i=1, \ldots, n, f_{i} \in \mathcal{K}_{1}^{*} \cup\{0\}$ and $0^{*}=0$;

- if $A$ is maxitive, i.e., if $A(\mathbf{x})=\max _{i} f_{i}\left(x_{i}\right)$ then $A^{*}$ is modular, $A^{*}(\mathbf{x})=\sum_{i=1}^{n} f_{i}^{*}\left(x_{i}\right)$, where for $i=1, \ldots, n, f_{i} \in \mathcal{K}_{1}^{*} \cup\{0\}$ and $0^{*}=0$;

- for $A \in \mathcal{K}_{n}^{*}$, and $\left.c \in\right] 0, \infty\left[\right.$, define $A_{c}: \mathbb{R}_{+}^{n} \rightarrow \mathbb{R}_{+}$by $A_{c}(\mathbf{x})=c A\left(\frac{\mathbf{x}}{c}\right)$. Then $A_{c} \in \mathcal{K}_{n}^{*}$ and $\left(A_{c}\right)^{*}=\left(A^{*}\right)_{c}$;

- for the power root operators $A_{p}: \mathbb{R}_{+}^{n} \rightarrow \mathbb{R}_{+}^{n}$ given by $A_{p}\left(x_{1}, \ldots, x_{n}\right)=\left(\frac{1}{n} \sum_{i=1}^{n} x_{i}^{p}\right)^{1 / p}, p \in \mathbb{R} \backslash\{0\}$, it holds $A_{p}^{*}=A_{p}$ if and only if $p \leq 1$, while, for $p>1$, we have $A_{p}^{*}\left(x_{1}, \ldots, x_{n}\right)=\frac{1}{n^{1 / p}} \cdot \sum_{i=1}^{n} x_{i}$;

- for $D$ introduced in Section 2, $D \notin \mathcal{K}_{2}^{*}$.

In the case of aggregation on $\mathbb{R}_{+}^{2}, A, B$ and $C$ being aggregation functions, it can be shown that for composite functions $A(B, C)^{*} \leq A^{*}\left(B^{*}, C^{*}\right)$ (due to monotonicity), but the equality need not hold. For example, consider $A(x, y)=x+y, B(x, y)=$ $x^{2}+y^{2}, C(x, y)=g(x)+g(y)$, where $g:[0, \infty[\rightarrow[0, \infty[$ is given by

$$
g(x)= \begin{cases}2 x-x^{2} & \text { if } x \leq 1 \\ 1 & \text { else }\end{cases}
$$

then $A^{*}=A, B^{*}=B, C^{*}(x, y)=2 x+2 y$, i.e., $A(B, C)^{*}(x, y)=\min \left(2 x, x^{2}+1\right)+$ $\min \left(2 y, y^{2}+1\right)<A^{*}\left(B^{*}, C^{*}\right)(x, y)=2 x+2 y+x^{2}+y^{2}$.

Interesting is also, on $\mathbb{R}_{+}^{3}$, the following relation between median, minimum and maximum:

$$
\operatorname{Med}^{*}(x, y, z)=\min \left\{\frac{x+y+z}{2}, \min (x, y, z)+\operatorname{Med}(x, y, z)\right\} .
$$

Remark 3 Observe that $\left(\mathcal{K}_{n}^{*}, \leq\right)$ is a lattice and the superadditive transformation is a closure operator on it.

Remark 4 Consider a homogeneous aggregation function $A \in \mathcal{K}_{n}^{*}$. It is not difficult to check that then also $A^{*}$ is homogeneous, and that it is the smallest superlinear aggregation function dominating $A$. Moreover, there exists $W \subseteq \mathbb{R}_{+}^{n}$ such that

$$
A^{*}(\boldsymbol{x})=\min \left\{\sum_{i=1}^{n} x_{i} w_{i}: \boldsymbol{w} \in W\right\}
$$


Indeed, it is well known (see [5], [1]) that a function $B: \mathbb{R}^{n} \rightarrow \mathbb{R}$ is superlinear if and only if there exists a compact and convex subset $C$ of $\mathbb{R}_{+}$such that

$$
B(x)=\min \left\{\sum_{i=1}^{n} x_{i} c_{i}: c \in C\right\}
$$

for all $x \in C$. Moreover $C$ is unique.

Observe that the median operator Med is a homogeneous aggregation function from $\mathcal{K}_{n}^{*}$. After a short processing of formula (4), we have an equivalent expression for $\operatorname{Med}^{*}(x, y, z)$, namely

$$
\left.\operatorname{Med}^{*}(x, y, z)=\min \left\{\frac{x+y+z}{2}, x+y, x+z, y+z\right)\right\},
$$

i.e., the set $W$ of weighting vectors related to the ternary median operator is given by $W=\{(0.5,0.5,0.5),(1,1,0),(1,0,1),(0,1,1)\}$, and the related compact convex set $C$ is just the convex closure of $W$.

\section{Subadditive transformation of aggregation functions}

In defining superadditive transformation of an aggregation function $A(\boldsymbol{x})$, we act from below, by looking for the supremum of sums $\sum_{j=1}^{k} A\left(\boldsymbol{y}^{j}\right), \sum_{j=1}^{k} \boldsymbol{y}^{j}$ being not greater than $\boldsymbol{x}$. We can reverse these passages, acting from above, and by looking for the infimum of sums $\sum_{j=1}^{k} A\left(\boldsymbol{y}^{j}\right), \sum_{j=1}^{k} \boldsymbol{y}^{j}$ being not smaller than $\boldsymbol{x}$. Although some results are quite similar to those of previous Section 3, for the sake of completeness, we propose them here.

Definition 2 Given an aggregation function $A$ on $\mathbb{R}_{+}^{n}$ and $\boldsymbol{x} \in \mathbb{R}_{+}^{n}$, if

$$
\inf \left\{\sum_{j=1}^{k} A\left(\boldsymbol{y}^{j}\right): \boldsymbol{y}^{j} \in \mathbb{R}_{+}^{n} \text { such that } \sum_{j=1}^{k} \boldsymbol{y}^{j} \geq \boldsymbol{x}\right\}>0, \quad \text { (pos) }
$$

then the subadditive transformation of $\boldsymbol{x}$ with respect to $A$ is given by

$$
A_{*}(\boldsymbol{x})=\inf \left\{\sum_{j=1}^{k} A\left(\boldsymbol{y}^{j}\right): \boldsymbol{y}^{j} \in \mathbb{R}_{+}^{n} \text { such that } \sum_{j=1}^{k} \boldsymbol{y}^{j} \geq \boldsymbol{x}\right\} .
$$

If (pos) is is not satisfied then $A_{*}(\boldsymbol{x})=0$.

Condition (pos) is a non-triviality condition, to avoid that $A_{*}(\boldsymbol{x})$ collapses into zero. Take, for example, the product $\Pi$, or the minimum min, we have that the inf in condition (pos) is zero, and then these aggregation functions do not yield an aggregation function $A_{*}$. However, similarly as in the case of Theorem 1, one can show that if

$$
\inf \left\{\sum_{j=1}^{k} A\left(\boldsymbol{y}^{j}\right): \boldsymbol{y}^{j} \in \mathbb{R}_{+}^{n} \text { such that } \sum_{j=1}^{k} \boldsymbol{y}^{j} \geq \mathbf{1}_{N}\right\}=0
$$


then

$$
\inf \left\{\sum_{j=1}^{k} A\left(\boldsymbol{y}^{j}\right): \boldsymbol{y}^{j} \in \mathbb{R}_{+}^{n} \text { such that } \sum_{j=1}^{k} \boldsymbol{y}^{j} \geq \boldsymbol{x}\right\}=0
$$

for any $\boldsymbol{x} \in \mathbb{R}_{+}^{n}$.

We say that an aggregation function $A$ on $\mathbb{R}_{+}^{n}$ admits subadditive transformation if condition (pos) is satisfied for some (at least one) $\boldsymbol{x} \in \mathbb{R}_{+}^{n}$. Due to the above observations it is evident that $A$ admits subadditive transformation if and only if $A_{*}\left(\mathbf{1}_{N}\right)>0$. We denote the class of all such aggregation functions on $\mathbb{R}_{+}^{n}$ by $\mathcal{K}_{*}^{n}$.

Next Proposition 3 represents a characterization of subadditive aggregation functions, by establishing that $A_{*}$ is the greatest subadditive aggregation function not greater than $A$. For the proof, the reader can follow the line of proof of Propositions 1 and 2.

Proposition 3 For any aggregation function $A$ on $\mathbb{R}_{+}^{n}$,

- $A_{*} \leq A$,

- $A_{*}(\mathbf{0})=0$, and

- $A_{*}(\boldsymbol{x}) \geq A_{*}(\boldsymbol{y})$ for all $\boldsymbol{x}, \boldsymbol{y} \in \mathbb{R}_{+}^{n}$ such that $\boldsymbol{x} \geq \boldsymbol{y}$,

- $A_{*}$ is subadditive.

Moreover

$$
A_{*}(\boldsymbol{x})=\max \left\{C(\boldsymbol{x}): C(\boldsymbol{y}) \leq A(\boldsymbol{y}), \forall \boldsymbol{y} \in \mathbb{R}_{+}^{n}\right\},
$$

where the maximum is taken over all the subadditive aggregation functions on $\mathbb{R}_{+}^{n}$ bounded from above by $A$.

Now we are ready to introduce a subadditive transformation $\tau_{*}$ on $\mathcal{K}_{*}^{n}$

Theorem 4 Define a mapping $\tau_{*}: \mathcal{K}_{*}^{n} \rightarrow \mathcal{K}_{*}^{n}$ by $\tau_{*}(A)=A_{*}$. Then $\tau_{*}$ is a subadditive transformation.

Proof. Due to Proposition 3, $\tau_{*}(A)$ is an aggregation function on $\mathbb{R}_{+}^{n}$ for any $A \in \mathcal{K}_{*}^{n}$. Moreover, $\left(A_{*}\right)_{*}\left(\mathbf{1}_{N}\right)=A_{*}\left(\mathbf{1}_{N}\right)$ and thus $\tau_{*}(A) \in \mathcal{K}_{*}^{n}$, i.e., $\boldsymbol{\tau}_{*}$ is well defined. It is not difficult to check that $\tau_{*}(A)=A_{*}$ is subadditive and $\left(A_{*}\right)_{*}=A_{*}$. Moreover, each subadditive aggregation function $A \in \mathcal{K}_{*}^{n}$ and $\tau_{*}(A)=A$ (proof is similar as in Theorem 3). Summarizing, $\tau_{*}$ is a subadditive transformation on $\mathcal{K}_{*}^{n}$.

Recall that if $A \notin \mathcal{K}_{*}^{n}$ then there is no subadditive aggregation function $B$ on $\mathbb{R}_{+}^{n}$ dominated by $A$ (formally, then $A_{*} \equiv 0$ ). We list some properties and examples:

- $A$ is subadditive if and only if $A_{*}=A$;

- $A_{* *}=A_{*}$;

- if $A \geq B$ then $A_{*} \geq B_{*}$;

- if $A \geq B \geq C$ and $A_{*}=C_{*}$, then $B_{*}=A_{*}$;

- if $A \geq B \geq D, A \geq C \geq D$, and $A_{*}=D_{*}$, then

$(\lambda B+(1-\lambda) C)_{*}=A_{*}$ for any $\lambda \in[0,1]$;

- $\operatorname{Max}_{*}=\operatorname{Max}$ (i.e., Max is subadditive); 
- if $A$ is modular, i.e., if $A(\mathbf{x})=\sum_{i=1}^{n} f_{i}\left(x_{i}\right)$ then $A_{*}$ is modular, $A_{*}(\mathbf{x})=\sum_{i=1}^{n}\left(f_{i}\right)_{*}\left(x_{i}\right)$, where for $i=1, \ldots, n, f_{i} \in \mathcal{K}_{*}^{1} \cup\{0\}$ and $0_{*}=0$;

- if $A$ is maxitive, i.e., if $A(\mathbf{x})=\max _{i} f_{i}\left(x_{i}\right)$ then $A_{*}$ is also maxitive, $A_{*}(\mathbf{x})=\operatorname{Max}_{i}\left(f_{i}\right)_{*}\left(x_{i}\right)$, where for $i=1, \ldots, n, f_{i} \in \mathcal{K}_{*}^{1} \cup\{0\}$ and $0_{*}=0$;

- for $\left.A \in \mathcal{K}_{*}^{n}, c \in\right] 0, \infty\left[\right.$, also $A_{c} \in \mathcal{K}_{*}^{n}$ (see Section 4). Then $\left(A_{c}\right)_{*}=\left(A_{*}\right)_{c}$;

- for the power root operators, $A_{p} \in \mathcal{K}_{*}^{n}$ if and only if $p>0$; if $\left.\left.p \in\right] 0,1\right]$, then $\left(A_{p}\right)_{*}(\boldsymbol{x})=\frac{1}{n^{1 / p}} \sum_{i=1}^{n} x_{i}$, while for $p \geq 1$ it holds $\left(A_{p}\right)_{*}=A_{p}$;

- the ternary median Med does not admit a subadditive transformation, Med $\notin \mathcal{K}_{*}^{3}$;

- for $D$ introduced in Section 2, $D \in \mathcal{K}_{*}^{2}$ and $D(x, y)=\sqrt{y}$.

Remark 5 Observe that the subadditive transformation is a closure operator on the lattice $\left(\mathcal{K}_{*}^{n}, \geq\right)$.

Remark 6 Consider a homogeneous aggregation function $A \in \mathcal{K}_{*}^{n}$. It is not difficult to check that then also $A_{*}$ is homogeneous, and that it is the greatest convex aggregation function dominated by $A$. Note that $A_{*}$ is sublinear. Moreover, analogous to what happens with superadditive transformation, there exists $W \subseteq \mathbb{R}_{+}^{n}$ such that

$$
A_{*}(\boldsymbol{x})=\max \left\{\sum_{i=1}^{n} x_{i} w_{i}: \boldsymbol{w} \in W\right\}
$$

Indeed, it is wellknown (see [5]) that a function $B: \mathbb{R}^{n} \rightarrow \mathbb{R}$ is sublinear if and only if there exists a compact and convex subset $C$ of $\mathbb{R}_{+}$such that

$$
B(x)=\max \left\{\sum_{i=1}^{n} x_{i} c_{i}: \boldsymbol{c} \in C\right\}
$$

for all $x \in C$. Moreover $C$ is unique.

\section{Superadditive transformations of integrals}

All integrals introduced in Section 2 admit superadditive transformation. This fact follows from Theorem 1. Indeed denoting any of the introduced integrals with respect to a measure $\mu$ as $I_{\mu}$ it holds $I_{\mu}\left(\mathbf{1}_{N}\right) \leq n \cdot \mu(N)<+\infty$. Moreover, as noted in [9], the concave integral is homogeneous and concave, hence also superadditive, and thus $\left(\int^{c a v} \boldsymbol{x} d \mu\right)^{*}=\int^{c a v} \boldsymbol{x} d \mu$, i.e., the concave integral is invariant under the superadditive transformation $\tau^{*}$.

Next Theorem 5 and the following Corollary 4 establish that each integral bounded from above by the concave integral and greater than the Shilkret integral, admits the concave integral as superadditive transformation.

Theorem 5 The concave integral is the superadditive extension of the Shilkret integral, i.e., $\left(\int^{S h} \boldsymbol{x} d \mu\right)^{*}=\int^{c a v} \boldsymbol{x} d \mu$. 
Proof. By definition $\int^{S h} \boldsymbol{x} d \mu \leq \int^{c a v} \boldsymbol{x} d \mu$ and since the concave integral is superadditive, $\left(\int^{S h} \boldsymbol{x} d \mu\right)^{*} \leq \int^{c a v} \boldsymbol{x} d \mu$. Moreover, it holds

$$
\begin{array}{r}
\iint^{c a v} \boldsymbol{x} d \mu=\sup \left\{\sum_{A \subseteq N} \alpha_{A} \mu(A) \mid \sum_{A \subseteq N} \alpha_{A} \mathbf{1}_{A}=\boldsymbol{x}\right\}= \\
\sup \left\{\sum_{A \subseteq N} \int^{S h} \alpha_{A} \mathbf{1}_{A} d \mu \mid \sum_{A \subseteq N} \alpha_{A} \mathbf{1}_{A}=\boldsymbol{x}\right\} \leq\left(\int^{S h} \boldsymbol{x} d \mu\right)^{*} .
\end{array}
$$

Corollary 4 Given a measure $\mu: 2^{N} \rightarrow \mathbb{R}_{+}$, for each integral $I_{\mu}$ (or aggregation function) such that $\int^{S h} d \mu \leq I_{\mu} \leq \int^{c a v} d \mu$, we have that $I_{\mu}{ }^{*}=\int^{c a v} d \mu$.

Remark 7 Observe that there are many integrals between the Shilkret and the concave integrals, for example the Choquet and the PAN integrals. For several other examples see [15], [11]. For all these integrals, due to Corollary 4, their $\tau^{*}$-transform gives the corresponding concave integral.

Next Theorem 6 shows that also the Sugeno integral admits the concave integral as superadditive extension, but with respect to a transformed measure.

Theorem 6 Let $\nu$ be a measure, and consider a transformed measure $\mu_{\nu}$ defined by

$$
\mu_{\nu}(T)=\left\{\begin{array}{ll}
1 & \text { if } \nu(T)>0 \\
0 & \text { if } \nu(T)=0
\end{array},\right.
$$

then we have that $\left(\int^{S u} \boldsymbol{x} d \nu\right)^{*}=\int^{c a v} \boldsymbol{x} d \mu_{\nu}$.

Proof. Based on the definition of the Sugeno integral, for any measure $\nu$ and any positive constant $c \in] 0, \infty\left[, \int^{S u} \boldsymbol{x} d c \nu=c \int^{S u} \frac{\boldsymbol{x}}{c} d \nu\right.$, and thus $\left(\int^{S u} \boldsymbol{x} d c \nu\right)^{*}=$ $c\left(\int^{S u} \frac{\boldsymbol{x}}{c} d \nu\right)^{*}$. Consider first a normed measure (capacity, fuzzy measure) $\nu, \nu(N)=1$. Then

$$
\int^{S u} \boldsymbol{x} d \nu \leq \int^{S u} \boldsymbol{x} d \mu_{\nu} \leq \int^{c a v} \boldsymbol{x} d \mu_{\nu}
$$

and therefore

$$
\left(\int^{S u} \boldsymbol{x} d \nu\right)^{*} \leq\left(\int^{c a v} \boldsymbol{x} d \mu_{\nu}\right)^{*}=\int^{c a v} \boldsymbol{x} d \mu_{\nu}
$$

By definition

$$
\int^{c a v} \boldsymbol{x} d \mu_{\nu}=\sup \left\{\sum_{T \subseteq N} \alpha_{T} \mu_{\nu}(T) \mid \sum_{T \subseteq N} \alpha_{T} \mathbf{1}_{T} \leq \boldsymbol{x}\right\} .
$$


Given the nature of $\mu_{\nu}$, in the expression (6), we have the following equality between sums

$$
\sum_{T \subseteq N} \alpha_{T} \mu_{\nu}(T)=\sum_{\substack{T \subseteq N \\ \alpha_{T} \nu(T)>0}} \alpha_{T} .
$$

Now consider $\alpha_{T} \mathbf{1}_{T}$ such that $\alpha_{T} \nu(T)>0$ and decompose such vector into the $r-$ sum, $r \in \mathbb{N}$,

$$
\alpha_{T} \mathbf{1}_{T}=\frac{\alpha_{T}}{r} \mathbf{1}_{T}+\ldots+\frac{\alpha_{T}}{r} \mathbf{1}_{T}
$$

$r$ being large enough to be $\alpha_{T} / r<\nu(T)$. By definition $\int^{S u}\left(\alpha_{T} / r\right) \mathbf{1}_{T} d \nu=\alpha_{T} / r$. Thus, every $\alpha_{T}$ in every sum of type (7) can be expressed as sum of Sugeno integral w.r.t. $\nu$, and then $\int^{c a v} \boldsymbol{x} d \mu_{\nu} \leq\left(\int^{S u} \boldsymbol{x} d \nu\right)^{*}$, which conclude the proof for normed measures. If $\nu(N) \neq 1$, we put $c=\frac{1}{\nu(N)}$ and then $c \nu$ is a normed measure. The statement immediately follows from the homogeneity of concave integral and the equality $\mu_{c \nu}=\mu_{\nu}$.

\section{Subadditive transformations of integrals}

For each integral $I$ introduced in Section $2, I_{\mu}(\boldsymbol{x})=0$ whenever $\mu(\operatorname{supp} \boldsymbol{x})=0$, where the support supp $\boldsymbol{x}=\left\{i \in N \mid x_{i}>0\right\}$. Therefore, for a fixed measure $\mu$, if there is a partition $\left\{E_{1}, \ldots, E_{k}\right\}$ of $N$ such that $\mu\left(E_{1}\right)=\ldots=\mu\left(E_{k}\right)=0$ evidently $\left(I_{\mu}\right)_{*}\left(\mathbf{1}_{N}\right)=0$, and thus $I_{\mu} \notin \mathcal{K}_{*}^{n}$ (compare with the superadditive case, when $I_{\mu} \in \mathcal{K}_{*}^{n}$, independently of the integral $I$ and the measure $\mu$ ). Observe that measures with the above property do not dominate any subadditive measure. Therefore when considering the subadditive transformation of integrals, we will deal with measures dominating some subadditive measure only, and we will call such measures "strong measures".

Note that the convex integral is homogeneous and subadditive [10], and thus, for any strong measure $\mu,\left(\int^{c o n} \boldsymbol{x} d \mu\right)_{*}=\int^{c o n} \boldsymbol{x} d \mu$, i.e., the concave integral with respect to a strong measure $\mu$ is invariant under the subadditive transformation $\tau_{*}$.

For any integral $I$ satisfying $I_{\mu}\left(c \cdot 1_{E}\right)=c \cdot \mu(E)$ it is evident that $\left(I_{\mu}(\boldsymbol{x})\right)_{*} \leq \int^{c o n} \boldsymbol{x} d \mu$.

This holds, in particular, for the Shilkret and the Choquet integrals. Moreover, due to the fact that the Choquet integral is always dominating the convex integral, we have an immediate result.

Proposition 4 Let $\mu$ be a strong measure. Then, for any $\boldsymbol{x} \in \mathbb{R}_{+}^{n}$,

$$
\left(\int^{C h} \boldsymbol{x} d \mu\right)_{*}=\int^{c o n} \boldsymbol{x} d \mu .
$$

On the other hand, the subadditive transformation of the Shilkret integral can be strictly smaller than the convex integral. 
Example 1 Let $n=2$, i.e., $N=\{1,2\}$, and let the measure $\mu: 2^{N} \rightarrow \mathbb{R}^{+}$be given by $\mu(\{1\})=\mu(\{2\})=2, \mu(N)=3$.

Then $\mu$ is a strong measure, and for $\boldsymbol{x}=(1,2)$ we have

$\left(\int^{S h} \boldsymbol{x} d \mu\right)_{*}=\min \left\{\int^{S h} \boldsymbol{x} d \mu, \int^{S h}(1,0) d \mu+\int^{S h}(0,2) d \mu\right\}=\min \{4,2+4\}=4$, while

$\int^{\text {con }} \boldsymbol{x} d \mu=\min \{1 \cdot \mu(N)+1 \cdot \mu(\{2\}), 2 \cdot \mu(N), 1 \cdot \mu(\{1\})+2 \cdot \mu(\{2\})\}=$ $=\min \{3+2,6,2+4\}=5$

We have the next analogues of Theorem 5 and Corollary 4, whose proofs are trivial and therefore omitted.

Theorem 7 For a fixed strong measure $\mu: 2^{N} \rightarrow \mathbb{R}_{+}$, define a mapping $A_{\mu}: \mathbb{R}_{+}^{n} \rightarrow$ $\mathbb{R}_{+}$by $A_{\mu}(\boldsymbol{x})=\left(\max _{i} x_{i}\right) \cdot \mu(\operatorname{supp} \boldsymbol{x})$. Then $A_{\mu} \in \mathcal{K}_{*}^{n}$, and $\left(A_{\mu}\right)_{*}(\boldsymbol{x})=\int^{\text {con }} \boldsymbol{x} d \mu$.

Corollary 5 Let $\mu$ be a fixed strong measure on $N$. For any aggregation function $A$ satisfying $\int^{\text {con }} \boldsymbol{x} d \mu \leq A(\boldsymbol{x}) \leq A_{\mu}(\boldsymbol{x})$ for all $\boldsymbol{x} \in \mathbb{R}_{+}^{n}$, it holds $A \in \mathcal{K}_{*}^{n}$, and $A_{*}(\boldsymbol{x})=$ $\int^{\text {con } x} d \mu$.

Recall that the Choquet integral satisfies the constraints from Corollary 5, as well as several integrals introduced and discussed in [11] see also [8] .

Concerning the Sugeno integral, we have the next interesting result.

Proposition 5 Let $\mu: 2^{N} \rightarrow \mathbb{R}_{+}$be a measure. Then the Sugeno integral with respect to $\mu$ is subadditive, and thus $\left(\int^{S u} \boldsymbol{x} d \mu\right)_{*}=\int^{S u} \boldsymbol{x} d \mu$ for all $\boldsymbol{x} \in \mathbb{R}_{+}^{n}$, if and only if $\mu$ is subadditive.

Proof. The sufficiency of the subadditivity for the Sugeno integral to get the subadditivity of a measure immediately follows from the properties of the Sugeno integral. We must prove that the subadditivity of the Sugeno integral is also a necessary condition for the subadditivity of the measure. Let $\mu$ be a subadditive measure, we have to show that for any $\boldsymbol{x}, \boldsymbol{y} \in \mathbb{R}_{+}^{n}$,

$$
\int^{S u}(\boldsymbol{x}+\boldsymbol{y}) d \mu \leq \int^{S u} \boldsymbol{x} d \mu+\int^{S u} \boldsymbol{y} d \mu .
$$

Denote $a=\int^{S u} \boldsymbol{x} d \mu, b=\int^{S u} \boldsymbol{y} d \mu$. Then $\mu(\{\boldsymbol{x} \geq a+\varepsilon\}) \leq a$ and $\mu(\{\boldsymbol{y} \geq b+\varepsilon\}) \leq b$ for each $\varepsilon>0$, and thus $\mu(\{\boldsymbol{x}+\boldsymbol{y} \geq a+b+2 \varepsilon\}) \leq \mu(\{\boldsymbol{x} \geq a+\varepsilon\} \cup\{\boldsymbol{y} \geq b+\varepsilon\}) \leq$ $\mu(\{\boldsymbol{x} \geq a+\varepsilon\})+\mu(\{\boldsymbol{y} \geq b+\varepsilon\}) \leq a+b$, where the second inequality follows from the subadditivity of $\mu$. Consequently, $\int^{S u}(\boldsymbol{x}+\boldsymbol{y}) d \mu \leq a+b$, proving the subadditivity of the discussed Sugeno integral.

In general, for any strong measure $\mu$, there is the greatest subadditive measure $\mu_{*}$ dominated by $\mu$. Indeed, $\mu_{*}: 2^{N} \rightarrow \mathbb{R}_{+}$is given by

$$
\mu_{*}(E)=\min \left\{\sum_{i=1}^{k} \mu\left(E_{i}\right) \mid\left\{E_{1}, \ldots, E_{k}\right\} \text { is a partition of } E\right\} .
$$


Obviously, $\left(\int^{S u} \boldsymbol{x} d \mu\right)_{*} \geq \int^{S u} \boldsymbol{x} d \mu_{*}$ for each $\boldsymbol{x} \in \mathbb{R}_{+}^{n}$. We conjecture that even the equality holds, i.e., $\left(\int^{S u} \boldsymbol{x} d \mu\right)_{*}=\int^{S u} \boldsymbol{x} d \mu_{*}$, in general. Observe that if $n=2$, our conjecture is true.

\section{Concluding remarks}

We have introduced and studied superadditive and subadditive transformations of ( $n$ ary) aggregation functions on $\mathbb{R}_{+}$. Interestingly, these transformations relate several distinguished integrals. So, for example, the superadditive transformation of the Choquet (or of the Shilkret) integral yields just the related concave integral based on the same measure. In the case of Sugeno integral, its superadditive transformation remains the Sugeno integral, but with respect to a transformed measure. Similar results are valid for subadditive transformations (in the case of Sugeno integral, we have formulated a conjecture; note that this conjecture is true for $n=2$ ).

Our approach is related in some particular cases with the integral with respect to a fuzzy capacity proposed by Lehrer in [9]. Indeed, for any non-decreasing positively homogeneous function $\nu:[0,1]^{n} \rightarrow \mathbb{R}_{+}, \nu\left(\mathbf{1}_{N}\right)>0$, it is evident that the function $A_{\nu}: \mathbb{R}_{+}^{n} \rightarrow \mathbb{R}_{+}$given by $A_{\nu}(\boldsymbol{x})=c \nu\left(\frac{\boldsymbol{x}}{c}\right)$, where $c=\max _{i} x_{i}, \boldsymbol{x} \neq(0, \ldots, 0)$, and $A_{\nu}(0, \ldots, 0)=0$, is a homogeneous aggregation function. Then $A_{\nu} \in \mathcal{K}_{n}^{*}$, and $A_{\nu}^{*}$ coincide with Lehrer's concave integral with respect to the fuzzy capacity $\nu$.

Note that our work can be considered as the theoretical background for constructing aggregation functions with given properties - in our case the super/subadditivity was considered. Our approach can be taken also as an optimization problem, with potential real applications. However, in real life situation, we often have only a partial information about the aggregation function $A$, i.e., $A$ is known only on a subdomain of $\mathbb{R}_{+}^{n}$. This is, for example, the case of measures which can be seen as instances of aggregation functions known on inputs from $\{0,1\}^{n}$ (subsets of $N$ are identified with the corresponding characteristic vectors). A deeper study of super/subadditive extensions in such cases will be a challenging topic for the further investigation.

\section{Acknowledgment}

The work on this contribution was partially supported by the grant VEGA 1/0420/15, and also by the "Programma Operativo Nazionale" Ricerca \& Competitivitá 2007-2013 within the project PON04a2 E SINERGREEN-RES-NOVAE.

We are grateful to two anonymous reviewers for their constructive comments, which helped us to improve the manuscript.

\section{References}

1. C. Aliprantis, and K. Border. Infinite Dimensional Analysis. Springer-Verlag, 1999.

2. G. Choquet. Theory of Capacities. Annales de l'Institute Fourier (Grenoble), 5:131-295, 1953/54. 
3. T. J. Coelli, D. S. P. Rao, C. J. O'Donnell, and G. E. Battese. An introduction to efficiency and productivity analysis. Springer Science \& Business Media, 2005.

4. M. Grabisch, J.-L. Marichal, R. Mesiar, and E. Pap. Aggregation Functions (Encyclopedia of Mathematics and its Applications). Cambridge University Press, 2009.

5. L. Hörmander. Sur la fonction d'appui des ensembles convexes dans un espace localement convexe. Arkiv för Matematik, 3(2):181-186, 1955.

6. G. A. Jehle and P. J. Reny. Advanced microeconomic theory. Pearson Education India, 2001.

7. E.P. Klement, R. Mesiar, and E. Pap. Triangular Norms. Kluwer Academic Publisher, 2000.

8. E.P. Klement, R. Mesiar, F. Spizzichino, and A. Stupňanová. Universal integrals based on copulas. Fuzzy Optimization and Decision Making, 13:273-286, 2014.

9. E. Lehrer. A new integral for capacities. Economic Theory, 39:157-176, 2009.

10. R. Mesiar, J. Li, and E. Pap. Superdecomposition integral. Fuzzy Sets and Systems, 259:3-11, 2015.

11. R. Mesiar and A. Stupňanová. Decomposition integrals. International Journal of Approximate Reasoning, 54(08):1252-1259, 2013.

12. S. K. Mishra. A brief history of production functions. The IUP Journal of Managerial Economics, 8(3):6-34, 2010.

13. David Shephard, R. W. Gale and Harold W Kuhn. Theory of cost and production functions. Number 4. D. Gale, \& H. W. Kuhn (Eds.). Princeton: Princeton University Press, 1970.

14. N. Shilkret. Maxitive measure and integration. In Indagationes Mathematicae (Proceedings), volume 74, pages 109-116. Elsevier, 1971.

15. A. Stupňanová. A Note on Decomposition Integrals. In Advances in Computational Intelligence, pages 542-548. Springer, 2012.

16. M. Sugeno. Theory of fuzzy integrals and its applications. Ph.D. Thesis, Tokyo Institute of Technology, 1974.

17. Z. Wang and G. Klir. Generalized measure theory, volume 25. Springer, New York, 2009.

18. Q. Yang. The pan-integral on the fuzzy measure space. Fuzzy Mathematics (in Chinese), 3:107-114, 1985. 